INPLASY

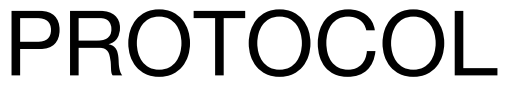

To cite: Yang et al. Dysphagia and Pneumonia in Acute Stroke Patients: A Systematic Review and Meta-analysis. Inplasy protocol 2021110108. doi:

10.37766/inplasy2021.11.0108

Received: 30 November 2021

Published: 30 November 2021

Corresponding author:

Min Cheol Chang

wheel633@ynu.ac.kr

Author Affiliation:

Yuengnam Univ.

Support: None.

Review Stage at time of this submission: Data analysis Completed but not published.

Conflicts of interest:

None declared.

\section{Dysphagia and Pneumonia in Acute Stroke Patients: A Systematic Review and Meta-analysis}

Yang, S1; Seo, KC2; Choo, YJ3; Chang, MC4.

Review question / Objective: Dysphagia is a common complication after stroke and it is one of the most significant factors for development of pneumonia. The aim of this study was to investigate the relationship between dysphagia and pneumonia in post-stroke patients. Using a PICO strategy, patients with acute stroke was the population, with presence or absence of dysphagia as the intervention and comparators. The main outcome of interests were the incidence of pneumonia and number of deaths attributable to post-stroke dysphagia.

Condition being studied: The prevalence of dysphagia after stroke is reported to be about $30-65 \%$. The presence of dysphagia in stroke patient may cause poor dietary intake, dehydration, malnutrition, and pulmonary complications, which relates to poor prognosis. It has been reported that stroke patients with dysphagia are at about 3- to 11-fold increase risk of having pneumonia and the prevalence of pneumonia is higher in patients with dysphagia when compared with those without dysphagia. Despite these previous studies suggesting that dysphagia after stroke can lead to a poor prognosis, to the best of our knowledge, a meta-analysis on this topic has not yet been performed.

INPLASY registration number: This protocol was registered with the International Platform of Registered Systematic Review and Meta-Analysis Protocols (INPLASY) on 30 November 2021 and was last updated on 30 November 2021 (registration number INPLASY2021110108).

\section{INTRODUCTION}

Review question / Objective: Dysphagia is a common complication after stroke and it is one of the most significant factors for development of pneumonia. The aim of this study was to investigate the relationship between dysphagia and pneumonia in post-stroke patients. Using a PICO strategy, patients with acute stroke was the population, with presence or absence of dysphagia as the intervention and 
comparators. The main outcome of interests were the incidence of pneumonia and number of deaths attributable to poststroke dysphagia.

Condition being studied: The prevalence of dysphagia after stroke is reported to be about $30-65 \%$. The presence of dysphagia in stroke patient may cause poor dietary intake, dehydration, malnutrition, and pulmonary complications, which relates to poor prognosis. It has been reported that stroke patients with dysphagia are at about 3- to 11-fold increase risk of having pneumonia and the prevalence of pneumonia is higher in patients with dysphagia when compared with those without dysphagia. Despite these previous studies suggesting that dysphagia after stroke can lead to a poor prognosis, to the best of our knowledge, a meta-analysis on this topic has not yet been performed.

\section{METHODS}

Participant or population: Acute stroke patients.

Intervention: Presence of dysphagia.

Comparator: Absence of dysphagia.

Study designs to be included: The design of the studies to be included in this systematic review and meta-analysis was not specific and a full range of designs was considered. However, reviews, case reports, commentaries, letters, and animal studies were excluded.

Eligibility criteria: We applied the following inclusion criteria for the selection of articles: (1) acute stroke patients were recruited for the study; (2) dysphagia was diagnosed in intervention group (dysphagia group), and was not diagnosed in comparator group (control group); (3) the development of pneumonia or number of deaths were evaluated in both groups. The exclusion criteria were as follows: (1) studies were not related to dysphagia, pneumonia, or mortality (2) reviews, case reports, commentaries, letters, and animal studies; and (3) study outcomes were not reported or insufficient.

Information sources: We systematically searched for relevant articles using Pubmed, Embase, the Cochrane library, and SCOPUS databases, up until November 2021. The following keywords were used in the search "deglutition disorders", "deglutition", "dysphagia", "swallowing", "deglutition pneumonia", and "aspiration pneumonia".

Main outcome(s): The development of pneumonia or number of deaths were evaluated in intervention group and comparator group.

Quality assessment / Risk of bias analysis: The methodological qualities of the studies were assessed using the Risk Of Bias In Non-randomized Studies-of Interventions (ROBINS-I) tool for the included nonrandomized controlled trials. Funnel plot and Egger's test were also used to assess publication bias.

Strategy of data synthesis: Two independent reviewers excluded articles after reading titles and abstracts (SKC AND SYY) and full-text assessments were done to reject those ones not fulfilling the inclusion criteria. The reviewers attempted to resolve any disagreement by consensus. If necessary, the opinion of a third reviewer was put into consideration to resolve the disagreement. All data were independently extracted independently by two reviewers (SYY and MCC) using a standard data collection form. Discrepancies were resolved through consensus and discussions with another investigator (SKC), if necessary. The following data were recorded using a table from each eligible article: (1) the name of the first author, (2) year of publication, (3) number of patients, (4) number of diagnosis of dysphagia, (5) the incidence of pneumonia and number of deaths.

Subgroup analysis: Not applicable.

Sensitivity analysis: The studies were excluded one by one, and then the meta- 
analysis was performed in the remaining studies.

Language: English.

Country(ies) involved: Republic of Korea.

Keywords: dysphagia; pneumonia; stroke; mortality; meta-analysis.

Contributions of each author:

Author 1 - Seoyon Yang - The author read, provided feedback and approved the final manuscript.

Email: seoyonyang@gmail.com

Author 2 - Kyung Cheon Seo - The author read, provided feedback and approved the final manuscript.

Author 3 - Yoo Jin Choo - The author read, provided feedback and approved the final manuscript.

Email: cyj361@hanmail.net

Author 4 - Min Cheol Chang - The author read, provided feedback and approved the final manuscript.

Email: wheel633@ynu.ac.kr 